\title{
MORFOGÊNESE E ESTRUTURA DE BRACHIARIA DECUMBENS EM RESPOSTA AO CORTE E ADUBAÇÃO NITROGENADA
}

\author{
MORPHOGENESIS AND STRUCTURE OF BRACHIARIA DECUMBENS SUBMITTED TO \\ CUTMANAGEMENT AND NITROGEN FERTILIZATION
}

\author{
Da Silva, T.C. ${ }^{1}$, Perazzo, A.F. ${ }^{2}$, Macedo, C.H.O. ${ }^{1}$, Batista, E.D. ${ }^{1}$, Pinho, R.M.A. ${ }^{1}$, \\ Bezerra, H.F.C. ${ }^{1}$ e Santos, E.M. ${ }^{1}$
}

1'Departamento de Zootecnia. Universidade Federal de Viçosa. Viçosa-MG. Brasil. timao22@hotmail.com ${ }^{2}$ Departamento de Zootecnia. Universidade Federal da Paraíba. Areia-PB. Brasil.

\author{
PalaVRas chaVe adicionais \\ Comprimento da folha. Filocrono. Manejo. Número \\ de folhas. Perfilho.
}

\section{RESUMO}

Objetivou-se avaliar as características morfogênicas e estruturais do pasto de Brachiaria decumbens, submetido a intensidades, frequências de corte e adubação nitrogenada. $O$ delineamento experimental foi em blocos casualizados em esquema de parcelas subdivididas, tendo na parcela o arranjo fatorial $2 \times 2$ referente a combinações entre duas alturas de corte $(40$ e $50 \mathrm{~cm})$ e duas alturas de resíduo (15 e $25 \mathrm{~cm}$ ) e nas sub-parcelas a adubação nitrogenada (com ou sem nitrogênio), com quatro repetições. As variáveis analisadas para as características morfogênicas foram: taxa de alongamento foliar (TAIF); taxa de alongamento de colmo (TAIC); taxa de aparecimento foliar (TApF); taxa de senescência foliar (TSF) e filocrono; e para as características estruturais foram: número de perfilhos basais (NPB); número de folhas totais (NFT); número de folhas aparecidas (NFAp); número de folhas mortas (NFM); número de folhas vivas (NFV) e comprimento final da folha (CFF). A utilização de $100 \mathrm{~kg} / \mathrm{ha}$ de $\mathrm{N}$ permitiu aumento $(p<0,10)$ na TAIF de $31,58 \%$ e $126,32 \%$, respectivamente para os resíduos de 15 e $25 \mathrm{~cm}$. O NPB em comparação às alturas de corte $(40$ e $50 \mathrm{~cm})$ foi afetado $(p<0,10)$ apenas com a utilização da adubação nitrogenada, observando-se média de 145,62 perfilhos $/ \mathrm{m}^{2}$ para a altura de corte $50 \mathrm{~cm}$. A altura de resíduo $25 \mathrm{~cm}$ combinada com a altura de corte $40 \mathrm{~cm}$, junto à aplicação de $100 \mathrm{~kg} / \mathrm{ha}$ de $\mathrm{N}$, promovem maior produção de matéria seca total

Recibido: 01-10-10. Aceptado: 22-9-11.

\section{AdDitionAL KEYWORDS}

Leaf length. Phyllochron. Management. Number of leaves. Tiller.

em menor espaço de tempo (28 dias), permitindo maiores taxas de lotação e facilitando o manejo no sistema de lotação rotacionada.

\section{SUMMARY}

Aimed to evaluate the morphogenetic and structural characteristics of the pasture of Brachiaria decumbens, subjected to several intensities and frequencies of cutting and nitrogen fertilization. Experimental design was randomized blocks in split plots, whit a $2 \times 2$ factorial arrangement relating to combinations of two cutting heights $(40$ and $50 \mathrm{~cm}$ ) and two heights of residue ( 15 and $25 \mathrm{~cm}$ ) at the plots and nitrogen fertilization (with or without nitrogen) in sub-plots, with four replications. Mophogenetic variables evaluated were: leaf elongation rate (LER), stem elongation rate (SER), leaf appearance rate (LAR), leaf senescence rate (LSR) and phyllochron; and structural features were: number of basal tillers (NBT), total number of leaves (TNL), number of leaves appeared (NLAp), number of dead leaves (NDL), number of alive leaves (NAL) and final leaf length (FLL). $100 \mathrm{~kg} \mathrm{~N} /$ ha allowed increase $(p<0.10)$ on LER of $31.58 \%$ and $126.32 \%$ respectively for residues 15 and $25 \mathrm{~cm}$. The NBT compared to cutting heights $(40$ to $50 \mathrm{~cm}$ ) were affected $(p<0.10)$ only with the use of nitrogen fertilizer, with average of 145.62 tillers $/ \mathrm{m}^{2}$ in $50 \mathrm{~cm}$ cut height. Residue height of $25 \mathrm{~cm}$ combined with cut 
height $40 \mathrm{~cm}$ increase leaf elongation rate and number of basal tillers, reducing expansion time, without changing other structural characteristics of the pasture.

\section{INTRODUÇÃO}

Os pastos cultivados vêm ganhando espaço no Brasil em substituição aos nativos, principalmente devido ao padrão de produção que se firmou nos últimos anos, exigindo cultivares mais produtivas e adaptadas. De acordo com o Censo Agropecuário, realizado em 2006, dos 170 milhões de hectares de pastagens do país, cerca de 100 milhões de hectares são de pastagens cultivadas (IBGE, 2006).

O gênero Brachiaria tem destaque nas pastagens brasileiras, sendo o capim mais cultivado, pois apresenta vantagens a outros gêneros, como grande adaptabilidade a solos ácidos e de baixa fertilidade, no caso de algumas espécies como a Brachiaria decumbens, por exemplo, ao clima, além de proporcionar elevado rendimento de matéria seca (MS). Apesar da importância econômica deste gênero, constatam-se, com relativa frequência, falhas no sistema de produção dessas pastagens, pela adoção de técnicas e estratégias não adequadas (Moreira et al., 2009).

O nitrogênio é considerado um nutriente fundamental para a manutenção da produtividade e persistência de uma gramínea. É o principal constituinte das proteínas que participam ativamente na síntese dos compostos orgânicos que formam a estrutura do vegetal, desta forma, é responsável pelas características estruturais da planta (tamanho de folha, densidade de perfilho e folhas por perfilho), além de características morfogênicas (taxa de aparecimento foliar, taxa de alongamento foliar e senescência foliar) (Costa et al., 2006).

A adubação nitrogenada pode estimular a produtividade dos pastos, que pode variar quanto ao nível utilizado e a espécie. $\mathrm{O}$ suprimento de nitrogênio interfere na rebrota da forrageira após a desfolhação, pois altera as características e adaptações morfofisiológicas apresentadas pelas plantas na época (Alves et al., 2008). Além disso, proporciona um aumento na capacidade de suporte das pastagens, uma vez que acelera a formação e o crescimento de novas folhas e aumenta o vigor da rebrota.

O sucesso na utilização de pastagens não depende apenas da disponibilidade de nutrientes ou da escolha da espécie forrageira, mas também da compreensão dos mecanismos morfofisiológicos e de sua interação com o ambiente e do manejo.

A intensidade e freqüência de desfolhação é outro fator que influencia o acúmulo de biomassa. De acordo com Hodgson (1990), a intensidade de desfolhação indica a proporção do tecido vegetal removido pelo pastejo em relação ao disponibilizado para o pastejo.

$\mathrm{Na}$ lotação rotativa, a freqüência de desfolhação é determinada pela freqüência com os animais são movimentados de um piquete para outro, o que é função do tamanho do piquete, número de piquete, taxa de acúmulo líquido de forragem e número de animais. Assim, num determinado sistema, a duração média do período de descanso pode ser ajustada de forma a minimizar a perda de tecidos foliares devido a senescência, desde que a taxa de lotação e a duração do período de pastejo sejam suficientes para remover a máxima proporção da forragem acumulada (Nabinger, 2002).

A intensidade de desfolhação influencia a eficiência fotossintética das folhas nos primeiros estádios de rebrota, por isso, desfolhações intensas levam a menor eficiência inicial das folhas (Parsons et al., 1983). Esses autores alertam que quanto mais intensa a desfolhação, maior a taxa inicial de rebrota e maior o tempo necessário para que a planta atinja a máxima eficiência fotossintética e, conseqüentemente a máxima taxa de crescimento. Entretanto, a desfolhação muito severa pode esgotar as reservas de energia da planta e, assim, a densidade de perfilhos pode ser comprometida. 


\section{MORFOGÊNESE E ESTRUTURA DO BRACHIARIA DECUMBENS}

É provável que as características morfogênicas e estruturais de gramíneas de clima tropical sejam diferentes daquelas verificadas em regiões onde a estação chuvosa se concentra no verão pleno. $\mathrm{O}$ Brejo paraibano é uma microrregião com regime de chuvas concentrado no inverno, com temperaturas baixas, luminosidade reduzida, elevada umidade relativa do ar e precipitação pluviométrica em torno de 1400 $\mathrm{mm}$. A produção de forragem para ruminantes constitui, portanto, o maior desafio enfrentado pela pecuária explorada na região.

Objetivou-se neste trabalho avaliar a produção de matéria seca total, as características morfogênicas e estruturais do pasto de Brachiaria decumbens, submetido a intensidades, frequencia de corte e adubação nitrogenada.

\section{MATERIAL E MÉTODOS}

O experimento foi realizado no setor de Forragicultura do Centro de Ciências Agrárias (CCA), Campus II, da Universidade Federal da Paraíba (UFPB), localizado no município de Areia - PB, com latitude $6^{\circ} 57^{\prime}$ $46^{\prime \prime} \mathrm{S}$ e, uma longitude $35^{\circ} 41^{\prime} 31^{\prime \prime} \mathrm{W}$, e a 623 $\mathrm{m}$ de altitude. A temperatura média anual é de $22,3^{\circ} \mathrm{C}$, tendo 19,13 e $26,94^{\circ} \mathrm{C}$ como media mínima e máxima respectivamente e com umidade relativa média do ar de $82,04 \%$ (tabela I, dados obtidos na estação meteorológica da Universidade Federal da Paraíba). A espécie forrageira estudada foi a Bachiaria decumbens 'Basilisk'.
Utilizou-se uma área total de $218,08 \mathrm{~m}^{2}$, sendo dividida em 32 sub-parcelas de $6 \mathrm{~m}^{2}$ $(3 \times 2 \mathrm{~m})$ cada (unidades experimentais) formando a área utilizada de $192 \mathrm{~m}^{2}$. Cada subparcela foi avaliada numa área útil de $2 \mathrm{~m}^{2}$.

$\mathrm{O}$ delineamento experimental foi $\mathrm{em}$ blocos casualizados em esquema de parcelas subdivididas, tendo na parcela o arranjo fatorial $2 \times 2$ referente a combinações entre duas alturas de corte $(40$ e $50 \mathrm{~cm})$ e duas alturas de resíduo $(15$ e $25 \mathrm{~cm})$ e nas subparcelas a adubação nitrogenada (com ou sem N), com quatro repetições. Os tratamentos receberam as seguintes codificações: 15-50 (altura de resíduo de $15 \mathrm{~cm}$ combinada com altura de corte de $50 \mathrm{~cm}$ ); 25 50 (altura de resíduo de $25 \mathrm{~cm}$ combinada com altura de corte de $50 \mathrm{~cm}$ ); 15-40 (altura de resíduo de $15 \mathrm{~cm}$ combinada com altura de corte de $40 \mathrm{~cm}$ ) e 25-40 (altura de resíduo de $25 \mathrm{~cm}$ combinada com altura de corte de $40 \mathrm{~cm})$, com $(100 \mathrm{~kg} / \mathrm{ha}$ de N) ou sem adubação.

Foram coletadas amostras de solo na profundidade de $0-20 \mathrm{~cm}$, para as análises das características químicas, feitas no Laboratório de Solos do CCA-UFPB. O solo da área onde foi conduzido o experimento apresentou os seguintes atributos quími$\cos (0 \mathrm{a} 20 \mathrm{~cm}): \mathrm{pH}\left(\mathrm{H}_{2} \mathrm{O}\right)=5,28 ; \mathrm{P}=4,65, \mathrm{~K}^{+}$ $21,33 \mathrm{mg} / \mathrm{dm}^{3} ; \mathrm{Ca}^{+2}=1,30, \mathrm{Mg}^{+2}=0,35$, $\mathrm{CTC}=7,96, \mathrm{H}^{+}+\mathrm{Al}^{+3}=6,19, \mathrm{Al}^{+3}=0,45 \mathrm{cmol}_{\mathrm{c}} /$ $\mathrm{dm}^{3} ; \mathrm{MO}=19,43 \mathrm{~g} / \mathrm{kg}$.

Para a adubação de manutenção foram utilizados $100 \mathrm{~kg} / \mathrm{ha}$ de nitrogênio, segundo a recomendação para pastagens de nível

Tabela I. Médias climáticas registradas durante os meses de abril a setembro de 2009, na estação meteorológica da Universidade Federal da Paraíba, no município de Areia, Paraiba. (Climate averages recorded during months from April to September 2009, at the weather station at the Federal University of Paraíba, in Areia, Paraíba).

\begin{tabular}{lcccccc}
\hline Variáveis climáticas & Abril & Maio & Junho & Julho & Agosto & Setembro \\
\hline Temperatura média do ar ( $\left.{ }^{\circ} \mathrm{C}\right)$ & 23,5 & 22,7 & 21,6 & 21,0 & 20,8 & 21,6 \\
Precipitação pluvial (mm) & 245,2 & 271,4 & 212,2 & 319,6 & 183,4 & 40,4 \\
Umidade relativa do ar (\%) & 89 & 91 & 91 & 90 & 87 & 85 \\
Nebulosidade & 9,0 & 9,0 & 8,0 & 9,0 & 8,0 & 8,0 \\
\hline
\end{tabular}




\section{DA SILVA, PERAZZO, MACEDO, BATISTA, PINHO, BEZERRA E SANTOS}

tecnológico médio, segundo Cantarutti et al. (1999). O adubo foi aplicado na forma de sulfato de amônio, sendo que 50\% aplicado no dia 14 de Abril de 2009 (início do experimento) e o restante, trinta dias após a primeira.

O experimento foi realizado na estação chuvosa do ano de 2009, de abril a julho e foi conduzido até que cada tratamento atingisse sua altura de corte. Cada tratamento apresentou um período experimental e intervalo de cortes diferentes, sendo que nos tratamentos $15-50,25-50,15-40$ e $25-40$ sem adubação foi realizado apenas um com duração do cliclo de 103,103,103 e 98 dias respectivamente. Para os tratamentos 1550,25-50, 15-40 e 25-40 com adubação foram realizados $1,2,1$ e 2 cortes respectivamente com duração do ciclo de 45, 51, 45 e 28 dias respectivamente. Os cortes foram realizados quando pelo menos 3 subparcelas de um mesmo tratamento atingiam a altura de corte.

A altura do pasto foi medida com o uso de uma régua de disco adaptada com uma folha de transparência cortada circularmente ao centro, por onde passava um bastão de $2 \mathrm{~m}$ de comprimento graduado em centímetros. Foram tomadas leituras em pontos aleatórios por unidade experimental. A altura de cada ponto correspondeu à altura média do plano de folhas em torno da régua. As avaliações de altura foram realizadas sempre na condição pré-corte, quando as sub-parcelas atingiram alturas de 40 e/ou 50 $\mathrm{cm}$, e na condição pós-corte, as alturas residuais foram de $15 \mathrm{e} /$ ou $25 \mathrm{~cm}$.

Para a determinação da massa de forragem foram colhidas amostras representativas dos pastos nas condições de pré e pós-corte, utilizando duas armações de madeira de $0,5 \mathrm{~m}^{2}$ por unidade experimental. Após leitura da altura média do pasto com a régua de disco, as armações foram posicionadas em pontos representativos da altura média de corte cada tratamento no momento da amostragem e, as plantas contidas no interior de cada armação foram cortadas na altura de pré e pós-corte estabelecida, pesadas e secas em estufas a $65^{\circ} \mathrm{C}$ para determinação de matéria seca e componentes morfológicos.

As amostras de forragem foram pesadas e secas em estufa de circulação forçada de ar a $65^{\circ} \mathrm{C}$ até atingirem peso constante, para determinação do teor de matéria seca (MS). Os valores de massa de forragem foram convertidos para $\mathrm{kg} / \mathrm{ha}$ de MS multiplicando-se a produção de matéria verde pelo percentual de matéria seca da forragem.

Imediatamente após cada corte, foram marcados três perfilhos ao acaso por subparcela, os quais foram monitorados durante todo o período de rebrota. Após cada corte um novo grupo de três perfilhos foi marcado e o procedimento repetido até o final do experimento. Os perfilhos marcados foram identificados com fitas plásticas coloridas. Visando auxiliar a visualização no campo, foram fixados arames verticais com fita colorida, indicando a localização dos perfilhos. As avaliações nos perfilhos marcados foram realizadas a cada sete dias durante todo o período experimental, e consistiram da medição do comprimento das lâminas foliares, da altura da lígula da última folha expandida, da senescência foliar, além da contagem do número de novas folhas surgidas em cada um dos perfilhos. As variáveis analisadas para as características morfogênicas foram: taxa de alongamento foliar (TAlF); taxa de alongamento de colmo (TAlC); taxa de aparecimento foliar (TApF); taxa de senescência foliar (TSF) e filocrono; e para as características estruturais foram: número de perfilhos basais (NPB); número de folhas totais (NFT); número de folhas aparecidas (NFAp); número de folhas mortas (NFM); número de folhas vivas (NFV) e comprimento final da folha (CFF) segundo procedimento descrito por Marcelino et al. (2006).

Os dados foram submetidos à análise de variância e as médias comparadas pelo teste de Tukey ao nível de $10 \%$ de significância, utilizando-se o software SAEG, versão 8.0 (UFV, 1999). 


\section{RESULTADOSEDISCUSSÃO}

Na tabela II, observa-se que a interação altura de resíduo $\mathrm{x}$ altura de corte $\mathrm{x}$ adubação foi significativa $(\mathrm{p}>0,10)$ para a produção de matéria seca total (PMST). Observou-se diferença $(\mathrm{p}<0,10)$ entre as médias de intensidades e frequências de corte sem adubação, sendo que a menor média de PMST foi observada na altura de corte 40 cm combinada com a altura de resíduo de 15 $\mathrm{cm}$. Embora tenha apresentado maior valor, a PMST média observada para a intensidade e frequência de corte 25-40 não diferiu $(\mathrm{p}>0,10)$ das intensidades e frequências de 15-50 e 25-50. Estas, por sua vez, também não foram diferentes $(\mathrm{p}>0,10)$ da intensidade e frequência de corte 15-40.

Nas intensidades e frequências de corte com adubação, a maior $(\mathrm{p}<0,10)$ média de PMST foi de 5,24 t/ha, observada para intensidade e frequência de corte 25-50. Esse fato é explicado porque no pasto cortado a uma altura de $50 \mathrm{~cm}$ houve um maior tempo para acumular biomassa se comparado com as demais intensidades de corte, entretanto isto só foi observado para o resíduo $25 \mathrm{~cm}$, evidenciando que neste há uma maior reserva de carboidratos que possibilitam uma rebrota mais vigorosa. Para as intensidades e frequências de corte 15-40 e 25-40 observou-se PMST semelhante ( $p>0,10)$, ou menor $(p<0,10)$, no caso da intensidade e frequência de corte 15-50. Estudo feito por Matthew et al. (2000) ressalta que a altura de corte é importante, pois afeta a velocidade de rebrota, em razão da quantidade de tecido foliar fotossintetizante remanescente após o corte ou pastejo.

Deve-se salientar que a menor duração de ciclo (28 dias) foi observada na altura de corte $40 \mathrm{~cm}$ combinada com a altura de resíduo de $25 \mathrm{~cm}$. De posse dessa informação, é possível inferir que, mesmo PMST média inferior $(\mathrm{p}<0,10)$ à intensidade e frequência de corte $25-50$, altura de corte $40 \mathrm{~cm}$ combinada com o resíduo de $25 \mathrm{~cm}$ possibilitou
Tabela II. Produção de matéria seca total ( $t$ ) ha) de Brachiaria decumbens sob diferentes corte, resíduo, e adubação nitrogenada. (Total dry matter production (t/ha) of Brachiaria decumbens under several intensities and frequencies of cutting and nitrogen fertilization).

\begin{tabular}{lcccc}
\hline Altura de corte & \multicolumn{2}{c}{$50 \mathrm{~cm}$} & \multicolumn{2}{c}{$40 \mathrm{~cm}$} \\
Altura de resíduo & $15 \mathrm{~cm}$ & $25 \mathrm{~cm}$ & $15 \mathrm{~cm}$ & $25 \mathrm{~cm}$ \\
\hline $0 \mathrm{~kg} / \mathrm{ha}$ de $\mathrm{N}$ & $3,50^{\mathrm{ABa}}$ & $3,50^{\mathrm{ABb}}$ & $2,76^{\mathrm{Ba}}$ & $3,92^{\mathrm{Aa}}$ \\
$100 \mathrm{~kg} / \mathrm{ha}$ de $\mathrm{N}$ & $2,54^{\mathrm{Bb}}$ & $5,24^{\mathrm{Aa}}$ & $2,79^{\mathrm{Ba}}$ & $3,36^{\mathrm{Ba}}$ \\
\hline
\end{tabular}

${ }^{1}$ Médias seguidas de letras diferentes maiúsculas nas linhas e minúsculas nas colunas diferem entre si pelo teste de Tukey $(p<0,10)$. Coeficiente de variação $=18,40$.

uma maior produtividade média em menor intervalo de tempo, possibilitando maior rotatividade do pastejo.

Nas intensidades e frequências de corte 15-40 e 25-40, não houve efeito $(p>0,10)$ da adubação e no resíduo de $15 \mathrm{~cm}$ com altura de corte $50 \mathrm{~cm}$ a PMST média chegou a diminuir com a aplicação de adubo nitrogenado. Fagundes et al. (2006) observaram aumento na produção de matéria seca $(\mathrm{kg} /$ ha/ano) em função de doses crescentes de $\mathrm{N}(75,150,225$ e $300 \mathrm{~kg} / \mathrm{ha})$. Isso mostra o efeito da adubação nitrogenada, que quase chegou a aumentar em 50\% a PMST na intensidade e frequência de corte 25-50, de 3,50 para 5,24 t/ha. A adubação nitrogenada além de condicionar um aumento na produtividade das pastagens pode proporcionar um aumento na quantidade de proteína por hectare (Dias et al., 2000).

Estes resultados mostram que a adubação nitrogenada aumentou a velocidade de crescimento das plantas, principalmente nas com resíduo de $25 \mathrm{~cm}$, constatando o efeito do manejo sobre a eficiência de utilização do nitrogênio e ressaltando a necessidade de aplicação deste elemento para o aumento da produtividade em pastagens. Esse efeito pode ser atribuído à sua grande influência sobre os processos fisiológicos da planta 


\section{DA SILVA, PERAZZO, MACEDO, BATISTA, PINHO, BEZERRA E SANTOS}

(Herrera e Hernandez, 1985). Segundo Magalhães et al. (2007) a adubação nitrogenada promove aumento na relação folha:colmo, melhora o valor nutritivo e eleva a produtividade da forrageira.

Em condições edafoclimáticas, e mediante a ausência de outra limitação, seguramente o suprimento de $\mathrm{N}$ é o fator de maior impacto na produtividade da planta forrageira bem estabelecida e dos animais que a utilizam. Fagundes et al. (2006) conferiram que o suprimento de nitrogênio no solo normalmente não atende à demanda das gramíneas, porém, quando há adubação nitrogenada, são observadas grandes alterações na taxa de acúmulo de MS da forragem do capim-braquiária ao longo das estações do ano.

Foi observado efeito $(\mathrm{p}<0,10)$ das interações altura de resíduo $\mathrm{x}$ adubação e altura de corte $\mathrm{x}$ adubação para as variáveis TAlF e NPB (tabela III e tabela IV). Para as demais variáveis nenhuma das interações analisadas (altura de resíduo x adubação, altura de corte x adubação, altura de resíduo $\mathrm{x}$ altura de corte e altura de resíduo $\mathrm{x}$ altura de corte $x$ adubação) foi significativa $(\mathrm{p}>0,10)$. Para a maioria das variáveis

Tabela III. Taxa de alongamento foliar (TAlF) de Brachiaria decumbens em função de altura de corte, resíduo, e adubação nitrogenada. (Leaf elongation rate (LER) of Brachiaria decumbens under several intensities and frequencies of cutting and nitrogen fertilization).

\begin{tabular}{lcc}
\hline Altura de resíduo $(\mathrm{cm})$ & 15 & 25 \\
$0 \mathrm{~kg} / \mathrm{ha}$ de N & $0,19^{\mathrm{Ab}}$ & $0,19^{\mathrm{Ab}}$ \\
$100 \mathrm{~kg} / \mathrm{ha}$ de N & $0,25^{\mathrm{Ba}}$ & $0,43^{\mathrm{Aa}}$ \\
& & \\
Altura de corte $(\mathrm{cm})$ & 40 & 50 \\
$0 \mathrm{~kg} / \mathrm{ha}$ de N & $0,20^{\mathrm{Ab}}$ & $0,19^{\mathrm{Ab}}$ \\
$100 \mathrm{~kg} / \mathrm{ha}$ de N & $0,42^{\mathrm{Aa}}$ & $0,27^{\mathrm{Ba}}$ \\
\hline
\end{tabular}

ABabMédias seguidas de letras diferentes maiúsculas nas linhas e minúsculas nas colunas diferem entre si pelo teste de Tukey $(p<0,10)$. Coeficiente de variação $=15,20$. analisadas o comportamento de um fator em estudo não influenciou o outro fator, por isso, com exceção para TAlF e NPB, os fatores não sofreram nenhum desdobramento.

Na tabela III, observa-se que não houve efeito $(\mathrm{p}>0,10)$ na TAlF média entre as alturas de resíduo (15 e $25 \mathrm{~cm}$ ) sem a utilização da adubação nitrogenada. Por conseguinte, a utilização de $100 \mathrm{~kg} / \mathrm{ha}$ de $\mathrm{N}$ permitiu aumento $(\mathrm{p}<0,10)$ na TAlF de $31,58 \%$ e $126,32 \%$, respectivamente para os resíduos de 15 e $25 \mathrm{~cm}$. Em comparação aos tratamentos adubados observou-se maior TAlF $(p<0,10)$ para o resíduo de $25 \mathrm{~cm}$, com aumento de $72 \%$ em comparação ao resíduo de $15 \mathrm{~cm}$. Este aumento na TAlF para o maior resíduo pode estar associado devido ao $\mathrm{N}$ dar suporte a planta com maior reserva de carboidratos, para que a mesma emita folhas e complete o seu ciclo em um menor tempo, comparandoas com as que detém menor reserva, daí o entendimento quando se compara as duas alturas associadas com a utilização do N. Segundo Silveira et al. (2010), o processo de desenvolvimento e de expansão completa das folhas é determinado geneticamente e condicionado por variações nas condições

Tabela IV. Número de perfilhos basais de Brachiaria decumbens em função de altura de corte, resíduo e adubação nitrogenada. (Number of basal tillers of Brachiaria decumbens under several intensities and frequencies of cutting and nitrogen fertilization).

\begin{tabular}{lcc}
\hline Altura de resíduo $(\mathrm{cm})$ & 15 & 25 \\
$0 \mathrm{~kg} / \mathrm{ha}$ de $N$ & $99,87^{\mathrm{Ab}}$ & $91,37^{\mathrm{Ab}}$ \\
$100 \mathrm{~kg} / \mathrm{ha}$ de $\mathrm{N}$ & $120,25^{\mathrm{Aa}}$ & $125,17^{\mathrm{Aa}}$ \\
& & \\
Altura de corte $(\mathrm{cm})$ & 40 & 50 \\
$0 \mathrm{~kg} / \mathrm{ha}$ de $\mathrm{N}$ & $102,75^{\mathrm{Aa}}$ & $88,50^{\mathrm{Ab}}$ \\
$100 \mathrm{~kg} / \mathrm{ha}$ de N & $99,79^{\mathrm{Ba}}$ & $145,62^{\mathrm{Aa}}$ \\
\hline
\end{tabular}

ABabMédias seguidas de letras diferentes maiúsculas nas linhas e minúsculas nas colunas diferem entre si pelo teste de Tukey $(p<0,10)$. Coeficiente de variação= 16,15 . 
de ambiente. Os efeitos mais pronunciados são os relacionados com a temperatura e com o suprimento de nitrogênio.

Quando não foi realizada fertilização nitrogenada não foi observado efeito $(\mathrm{p}>0,10)$ na TAlF média para as alturas de corte de 40 e $50 \mathrm{~cm}$, por outro lado, as mesmas submetidas a adubação nitrogenada obtiveram respostas significativas $(\mathrm{p}<0,10)$ com acréscimo na TAlF de $110 \%$ e $42,10 \%$ para as alturas de 40 e $50 \mathrm{~cm}$ respectivamente, sendo a altura de corte de $40 \mathrm{~cm}$ o maior resultado, com TAlF média 55,55\% superior em relação a altura de corte de $50 \mathrm{~cm}$, em razão de atingir precocemente a altura desejada, caracterizando assim, um ciclo mais curto. Em um contexto geral os tratamentos com adubação nitrogenada se sobressaíram em relação aos não adubados. Marcelino et al. (2006) observaram efeito da altura de corte e do intervalo entre cortes no alongamento foliar do capim-marandu, em que a menor intensidade e o menor intervalo entre cortes acarretaram em maior alongamento foliar, devido à maior renovação dos tecidos, semelhante ao observado no presente trabalho. Mesmo sendo espécies diferentes dentro do mesmo gênero, a resposta observada para as intensidades e frequências de corte sobre o alongamento de folhas foi semelhante.

Nascimento Jr. et al. (2002), em um trabalho de revisão, discutiram que o uso de assimilados pelos meristemas foliares é determinado diretamente pela temperatura, e esta controla as taxas de divisão e expansão celular, além de criar uma demanda de C e N responsável por gerar energia e material para a expansão do tecido foliar. O nitrogênio tem efeito pronunciado sobre a taxa de alongamento de folhas, aspecto que pode estar relacionado com o grande acúmulo de N na zona de divisão celular (Gastal e Nelson, 1984). De acordo com Gastal et al. (1992), plantas deficientes em $\mathrm{N}$ apresentam reduções de três a quatro vezes nos valores de $\mathrm{TAlF}$ em relação àquelas mantidas sob condições não limitantes. Por isso, a TAlF pode representar aferidor de potencial de produção em uma análise comparativa de cultivares submetidos a um mesmo regime de cortes em determinado ambiente.

Na tabela IV, observa-se que as médias do número de perfilhos basais não diferiram ( $p>0,10)$ entre as alturas de resíduo ( 15 e 25 $\mathrm{cm})$ em função da utilização ou não da adubação nitrogenada. Entretanto, observou-se efeito $(\mathrm{p}<0,10)$ da adubação nitrogenada dentro de cada altura de resíduo, com aumento no número médio de perfilhos de 20,41\% e 36,99\% para as alturas de resíduo de 15 e $25 \mathrm{~cm}$ respectivamente. Provavelmente, as alturas de resíduo utilizadas no presente trabalho foram tão próximas a ponto de não interferir no perfilhamento, sendo este mais fortemente influenciado pela adubação nitrogenada. A adubação nitrogenada aumenta o fluxo de tecido e as taxas de aparecimento dos órgãos das plantas, inclusive dos perfilhos, de forma que a biomassa é produzida em uma menor escala de tempo. Silva et al. (2009), avaliando Brachiaria decumbens sob condições controladas em casa de vegetação, na região sudoeste da Bahia, observaram que o uso da adubação nitrogenada influenciou o número de perfilhos por planta, e as plantas que não receberam suprimento de nitrogênio praticamente não perfilharam. Alexandrino et al. (2004) também observaram incremento no perfilhamento da $B$. brizantha à medida que aumentaram a dose de nitrogênio.

Houve interação entre alturas de corte e adubação $(p<0,10)$ para o número de perfilhos basais (tabela IV). O número médio de perfilhos basais em comparação às alturas de corte $(40$ e $50 \mathrm{~cm})$ foram afetados $(p<0,10)$ apenas com a utilização da adubação nitrogenada, tendo a altura de corte $50 \mathrm{~cm}$, um acréscimo de $65,5 \%$ de perfilhos basais, com média de 145,62 perfilhos $/ \mathrm{m}^{2}$. Esse fato pode ser justificado pelo mecanismo de compensação tamanho densidade estudado em capim marandu por Sbrissia e Da Silva (2008), onde existe uma correlação linear negativa entre a densidade populacional de 


\section{DA SILVA, PERAZZO, MACEDO, BATISTA, PINHO, BEZERRA E SANTOS}

perfilhos e a massa de MS do perfilho, sendo a inclinação desta reta de correlação influenciada por variações no IAF, razão área foliar:volume e densidade aparente por perfilho. Dessa forma, a densidade populacional de perfilhos é o componente do IAF que permite a maior flexibilidade de ajuste por parte da planta a diferentes regimes de desfolha, razão pela qual o IAF é otimizado em pastos mantidos através de uma alta densidade populacional de perfilhos pequenos.

Dentro das alturas de corte, observouse efeito $(p<0,10)$ da adubação apenas para a altura de $40 \mathrm{~cm}$ com média de 102,75 perfilhos $/ \mathrm{m}^{2}$ para o tratamento sem adubação nitrogenada. Alexandrino et al. (2004), aplicando duas doses de nitrogênio, 275,0 e 500 $\mathrm{kg} / \mathrm{ha}$, observaram incremento no perfilhamento da $B$. brizantha à medida que aumentaram a dose. As plantas que não foram adubadas com nitrogênio apresentaram baixa taxa de perfilhamento (Silva $e t$ al., 2009). O resultado observado no presente trabalho difere dos trabalhos citados, onde o perfilhamento aumentou com adubação nitrogenada. Provavelmente, na altura de $40 \mathrm{~cm}$, o $\mathrm{N}$ aplicado foi direcionado para o alongamento de folhas e colmos possibilitando maior taxa de crescimento do dossel e consequentemente, uma maior frequência de cortes.

A tabela V, mostra que as variáveis NFT, NFAp, NFM, NFV e CFF, não foram afetadas $(\mathrm{p}>0,10)$ pela altura de resíduo $(15$ e $25 \mathrm{~cm})$ e altura de corte $(40$ e $50 \mathrm{~cm})$. Contudo quando avaliadas na presença da adubação nitrogenada, as variáveis NFT e NFV obtiveram um decréscimo de $21,83 \%$ e de $23,59 \%$ respectivamente com relação às mesmas não adubadas, ou seja, a dose de $\mathrm{N}$ $(100 \mathrm{~kg} / \mathrm{ha})$ reduziu de forma significativa a duração de vida das folhas. Carnevalli e Da Silva (1999) observaram que o NFV é um valor relativamente constante para uma dada espécie. No presente estudo, essa variável foi influenciada pela adubação nitrogenada, evidenciando a capacidade de adaptação das plantas a diferentes estratégias de manejo.

Com base nos resultados da tabela $\mathbf{V}$,

Tabela $\boldsymbol{V}$. Características estruturais de Brachiaria decumbens em função de altura de corte, resíduo, adubação nitrogenada. (Estructural characteristics of Brachiaria decumbens under several intensities and frequencies of cutting and nitrogen fertilization).

\begin{tabular}{lccccr}
\hline & NFT & NFAp & NFM & NFV & CFF \\
\hline Altura de resíduo & & & & & \\
15 & $9,44^{\mathrm{a}}$ & $4,90^{\mathrm{a}}$ & $3,36^{\mathrm{a}}$ & $6,16^{\mathrm{a}}$ & $13,67^{\mathrm{a}}$ \\
25 & $9,09^{\mathrm{a}}$ & $3,58^{\mathrm{a}}$ & $3,27^{\mathrm{a}}$ & $5,73^{\mathrm{a}}$ & $15,61^{\mathrm{a}}$ \\
$\begin{array}{l}\text { Altura de corte } \\
40\end{array}$ & $9,31^{\mathrm{a}}$ & $4,30^{\mathrm{a}}$ & $3,47^{\mathrm{a}}$ & $5,84^{\mathrm{a}}$ & $15,91^{\mathrm{a}}$ \\
$\quad 50$ & $9,22^{\mathrm{a}}$ & $4,18^{\mathrm{a}}$ & $3,16^{\mathrm{a}}$ & $6,05^{\mathrm{a}}$ & $13,77^{\mathrm{a}}$ \\
Adubo & $10,40^{\mathrm{a}}$ & $5,21^{\mathrm{a}}$ & $3,64^{\mathrm{a}}$ & $6,74^{\mathrm{a}}$ & $13,53^{\mathrm{b}}$ \\
$\quad \mathrm{kg} / \mathrm{ha}$ de N & $8,13^{\mathrm{b}}$ & $3,30^{\mathrm{b}}$ & $2,99^{\mathrm{a}}$ & $5,15^{\mathrm{b}}$ & $15,76^{\mathrm{a}}$ \\
$100 \mathrm{~kg} / \mathrm{ha} \mathrm{de} \mathrm{N}$ & 18,77 & 38,96 & 33,64 & 18,16 & 15,89 \\
CV $(\%)$ & & & & & \\
\hline
\end{tabular}

${ }^{a b}$ Médias seguidas de letras diferentes diferem entre si pelo teste de Tukey $(p<0,10)$.

NFT: Número de folhas totais (folhas/perfilho); NFAp: Número de folhas aparecidas (folhas/perfilho); NFM: Número de folhas mortas (folhas/perfilho); NFV: Número de folhas vivas (folhas/perfilho); CFF: Comprimento final de folha(mm/perfilho). CV: coeficiente de variação.

Archivos de zootecnia vol. 61, núm. 233, p. 98. 
pode-se inferir que as plantas, na ausência de adubação, permaneceram mais tempo com suas folhas vivas em detrimento da expansão de novas folhas, ou seja, o processo de senescência destas forrageiras é acelerado com aumento das doses de nitrogênio, reduzindo a duração de vida das folhas. Segundo Martuscello et al. (2005), a redução na duração de vida das folhas com a adubação nitrogenada pode ser explicada pela maior renovação de tecidos nas plantas.

A TAlC foi afetada $(p<0,10)$ apenas pela altura de corte, com maior média de 0,057 $\mathrm{mm} /$ perfilho/dia para a altura de $40 \mathrm{~cm}$, este aumento de $58,33 \%$ na TAlC em comparação ao resíduo de $50 \mathrm{~cm}$ pode estar associado á redução no ciclo da planta em função da menor altura de manejo.

O NFAp na presença da adubação nitrogenada também foi influenciado $(p<0,10)$, quando comparada com as mesmas sem a adubação, com decréscimo de $36,66 \%$ no NFAp (tabela V). Estes resultados podem estar relacionados com o efeito do nitrogênio na planta, que é observado principalmente sobre a TAlF, como observado no presente trabalho e por Garcez Neto et al. (2002). O alongamento foliar de gramíneas está restrito a uma zona na base da folha em expansão que está protegida pelo conjunto de bainhas das folhas mais velhas ou pseudocolmo. A TAlF é uma função do comprimento dessa zona de alongamento e da taxa de alongamento por segmento foliar, ou seja, das taxas de alongamento nas zonas de divisão celular (Skinner e Nelson, 1995). Por isso, como o alongamento de folhas é maior, provavelmente o número de folhas pode ser reduzido para manter o IAF ótimo (máxima interceptação luminosa).

$\mathrm{O}$ CFF, respondeu de forma positiva $(\mathrm{p}<0,10)$ ao suprimento da adubação nitrogenada, obtendo um acréscimo médio de $16,48 \%$ quando comparada com a mesma na ausência de N. Segundo Garcez Neto et al. (2002), quando as condições para o crescimento são favoráveis e constantes, a divisão celular é também favorecida, tornando possível obter lâminas maiores para mesmo comprimento de bainha. De acordo com esses autores, o aumento no tamanho de lâmina pode ser explicado pelo efeito simultâneo do N, que aumentou de forma expressiva o número de células em processo de divisão, e pela altura de corte, que determinou maior comprimento da bainha. O nitrogênio, ao estimular a produção de novas células, possibilita aumento na taxa de alongamento das folhas, o que pode constituir estratégia para mudanças no tamanho da lâmina foliar.

As características morfogênicas (TAlC, TApF, TSF e filocrono) verificadas na tabela VI não apresentaram efeito significativo em função das alturas de corte $(40$ e $50 \mathrm{~cm})$, entretanto, observou-se efeito $(p<0,10)$ da altura de resíduo $(15$ e $25 \mathrm{~cm}$ ) somente para TSF com maior taxa para o resíduo de $25 \mathrm{~cm}$ $(0,17 \mathrm{~mm} /$ perfilho/dia). Provavelmente, na menor altura de resíduo os tecidos apresentavam-se em pleno desenvolvimento e mais novos e com isso a sua taxa de senescência foi menor. Nas avaliações com a presença ou ausência de adubação nitrogenada, a TSF obteve maior resultado quando foi submetida à adubação nitrogenada, com TSF média de $0,20 \mathrm{~mm} /$ perfilho/dia, provavelmente por razão do $\mathrm{N}$ reduzir a duração de vida das folhas. Como discutido anteriormente, o nitrogênio atua principalmente na aceleração do fluxo e renovação dos tecidos, de forma que as folhas rapidamente se expandem e entram em processo de senescência mais rapidamente.

Os valores do filocrono apresentaram efeito $(\mathrm{p}<0,10)$ com relação ao suprimento de N no solo, observando-se menores valores para plantas que receberam a adubação nitrogenada (14,56 dias/folha), por motivo do elemento N potencializar o metabolismo da planta no que diz respeito à utilização dos carboidratos disponíveis, com isso aumentando a emissão de folhas e consequentemente diminuindo o tempo de aparecimento de uma folha pra a outra. 
DA SILVA, PERAZZO, MACEDO, BATISTA, PINHO, BEZERRA E SANTOS

Tabela VI. Características morfogênicas de Brachiaria decumbens em função de altura de corte, resíduo, e adubação nitrogenada. (Morphogenetic characteristics of Brachiaria decumbens under several intensities and frequencies of cutting and nitrogen fertilization).

\begin{tabular}{|c|c|c|c|c|}
\hline & TAIC & TApF & TSF & Filocrono \\
\hline \multicolumn{5}{|l|}{ Altura de resíduo } \\
\hline 15 & $0,045^{\mathrm{a}}$ & $0,079^{a}$ & $0,14^{b}$ & $15,95^{a}$ \\
\hline 25 & $0,048^{a}$ & $0,071^{a}$ & $0,17^{a}$ & $20,09^{a}$ \\
\hline \multicolumn{5}{|l|}{ Altura de corte } \\
\hline 40 & $0,057^{a}$ & $0,08^{\mathrm{a}}$ & $0,16^{a}$ & $18,82^{\mathrm{a}}$ \\
\hline 50 & $0,036 b$ & $0,07^{a}$ & $0,16^{a}$ & $17,23^{a}$ \\
\hline \multicolumn{5}{|l|}{ Adubo } \\
\hline $0 \mathrm{~kg} / \mathrm{ha}$ de $\mathrm{N}$ & $0,041^{a}$ & $0,067^{a}$ & $0,12^{\mathrm{b}}$ & $21,48^{a}$ \\
\hline $100 \mathrm{~kg} / \mathrm{ha}$ de $\mathrm{N}$ & $0,052^{\mathrm{a}}$ & $0,082^{\mathrm{a}}$ & $0,20^{a}$ & $14,56^{b}$ \\
\hline CV (\%) & 89,03 & 35,04 & 38,21 & 32,09 \\
\hline
\end{tabular}

Médias seguidas de letras diferentes diferem entre si pelo teste de Tukey $(p<0,10)$.

TAIC: Taxa de alongamento de colmo ( $\mathrm{mm} /$ perfilho.dia); TApF: Taxa de aparecimento de folhas (folhas/ perfilho.dia); TSF: Taxa de senescência de folhas (mm/perfilho.dia). CV: coeficiente de variação.

Alexandrino et al. (2004), avaliando o filocrono em $B$. brizantha, verificaram que, com o aumento das doses de nitrogênio, o filocrono reduziu de 12,20 para 6,99 dias, respectivamente, nas plantas adubadas com 0 a $20 \mathrm{~kg} / \mathrm{ha}$ de N. Silva et al. (2009) observaram redução do filocrono em Brachiaria decumbens de 8,76 para 7,35 dias, com a aplição de $100 \mathrm{~kg} /$ ha de N.

Os resultados observados no presente trabalho, assim como os relatados na literatura, evidenciam a importância do nitrogênio na redução do tempo para o aparecimento de duas folhas sucessivas, uma vez que aumenta a produção de novas células, que tem reflexo positivo no número de folhas por planta. Entretanto, deve-se destacar que os valores de filocrono, mesmo sob adubação nitrogenada, foram maiores do que os observados na literatura, o que está diretamente relacionado com as condições climáticas características da microrregião do Brejo paraibano. Isto por que o período chuvoso, que a princípio deveria ser o de maior desenvolvimento da gramínea, coincide com baixas temperaturas, elevada umidade relativa e alta nebulosidade. Estes fatores reduzem a velocidade de crescimento e afetam a quantidade e a qualidade da luz que é interceptada pelo dossel, e por esta razão as plantas mesmo com a adubação nitrogenada apresentaram maiores valores de filocrono em relação aos outros locais.

De posse das informações sobre as características morfogênicas e estruturais é possível estabelecer inter relações entre estas e as condições climáticas e as de manejo, conforme esquema descrito por Chapman e Lemaire (1993). Observou-se no presente trabalho que as características morfogênicas foram diretamente influenciadas pelas condições edafoclimáticas, principalmente pela fertilização nitrogenada. Quando adubadas com $\mathrm{N}$ as plantas apresentaram menor NFT, com maior CFF, devido ao aumento na TAlF. Como consequência direta, foi observada redução do filocrono, devido ao aumento da renovação de tecidos. Estas respostas fazem parte dos mecanismos que a gramínea tem de restabelecer a área foliar do dossel.

A altura de resíduo $25 \mathrm{~cm}$ combinada 


\section{MORFOGÊNESE E ESTRUTURA DO BRACHIARIA DECUMBENS}

com a altura de corte $40 \mathrm{~cm}$, junto à aplicação de $100 \mathrm{~kg} /$ ha de N, promovem maior produção de matéria seca total em menor espaço de

\section{BIBLIOGRAFIA}

Alexandrino, E., Nascimento Júnior, D., Mosquim, P.R., Regazzi, A.J. e Rocha, F.C. 2004. Características morfogênicas e estruturais na rebrotação da Brachiaria brizantha cv. Marandu submetida a três doses de nitrogênio. Rev Bras Zootecn, 33: 1372-1379.

Alves, J.S., Pires, A.J.V., Matsumoto, S.N., Figueiredo, M.P. e Ribeiro, G.S. 2008. Características morfológicas e estruturais da Brachiaria decumbens Stapf. submetida a diferentes doses de nitrogênio e volumes de água. Acta Vet Brasilica, 2: 1-10.

Carnevalli, R.A. e Da Silva, S.C. 1999. Validação de técnicas experimentais para avaliação de características agronômicas e ecológicas de pastagens de Cynodon dactylon cv. Coastcross1. Sci Agric, 56: 489-499.

Cantarutti, R.B., Martins, C.E., De Carvalho, M.M., Da Fonseca, D.M., Arruda, M.L., Vilela, H. e De Oliveira, F.T.T. 1999. Pastagens. Em: Ribeiro, A.C., P.T.G. Guimaraes e V.H. Alvarez V. (Eds.). Recomendação para o uso de corretivos e fertilizantes em Minas Gerais: $5^{a}$ aproximação. Comissão de Fertilidade do Solo do Estado de Minas Gerais. Viçosa, MG. pp. 331-341.

Chapman, D.F. and Lemaire, G. 1993. Morphogenetic and structural determinants of plant regrowth after defoliation. In: International grassland congress. Proceedings... SIR Publishing. Palmerston North. pp. 95-104.

Costa, N.L., Paulino, V.T. e Magalhães, J.A. 2006. Produção de forragem, composição química $e$ morfogênese de Panicum maximum cv. Vencedor sob diferentes níveis de adubação nitrogenada. Rev Cient Prod Anim, 8: 66-72.

Dias, P.F., Rocha, G.P., Rocha Filho, R.R., Leal, M.A.A., De Almeida, D.L. e Souto, S.M. 2000. Produção e valor nutritivo de gramíneas forrageiras tropicais, avaliadas no período das águas, sob diferentes doses de nitrogênio. Rev Ciên Agrotecnol, 24: 260-271.

Herrera, R.S. y Hernandez, Y. 1985. Efecto de la fertilización nitrogenada en la calidad de Cynodon dactylon cv. Coastcross-1. Rendi- tempo (28 dias), permitindo maiores taxas de lotação e facilitando o manejo no sistema de lotação rotacionada.

miento de matéria seca, proteína bruta y percentage de hojas. Pastos y Forrages, 8: 227-237.

Hodgson, J. 1990. Grazing management: science into pratice. John Wiley. Longman Scientific and Technical. New York. 203 pp.

Fagundes, L.J., Fonseca, D.M., Mistura, C., Morais, R.V., Vitor, C.M.T., Gomide, J.A., Nascimento Júnior, D., Casagrande, D.R. e Costa, L.T. 2006. Características morfogênicas e estruturais do capim-braquiária em pastagem adubada com nitrogênio avaliadas nas quatro estações do ano. Rev Bras Zootecn, 35: 21-29.

IBGE. 2006. Instituto Brasileiro de Geografia e Estatística. http://www.ibge.gov.br/home/ estatistica/economia/agropecuaria/censoagro/ brasil_2006/default.shtm (27/02/11).

Garcez Neto, A.F., Nascimento Júnior, D., Regazzi, A.J., Fonseca, D.M., Mosquim, P.R. e Gobbi, K.F. 2002. Respostas morfogênicas e estruturais de Panicum maximum cv. Mombaça sob diferentes níveis de adubação nitrogenada e alturas de corte. Rev Bras Zootecn, 31: 1890-1900.

Gastal, F. and Nelson, C.J. 1984. Nitrogen use within the growing leaf blade of tall fescue. Plant Physiol, 105: 191-197.

Gastal, F., Belanger, G. and Lemaire, G. 1992. A model of leaf extension rate of tall fescue in response to nitrogen and temperature. Ann Bot, 70: 437-442.

Magalhães, A.F., Pires, A.J.V., De Carvalho, G.G.P., Da Silva, F.F., Sousa, R.S. e Veloso, C.M. 2007. Influência do nitrogênio e do fósforo na produção do capim-braquiária. Rev Bras Zootecn, 36: 1240-1246.

Marcelino, K.R.A., Nascimento Júnior, D., Silva, S.C., Euclides, V.P.B. e Fonseca, D.M. 2006. Características morfogênicas e estruturais e produção de forragem do capim-marandu submetido a intensidades e freqüências de desfolhação. Rev Bras Zootecn, 35: 22432252.

Martuscello, J.A., Fonseca, D.M., Nascimento Júnior, D., Santos, P.M., Ribeiro Júnior, J.I., 


\section{DA SILVA, PERAZZO, MACEDO, BATISTA, PINHO, BEZERRA E SANTOS}

Cunha, D.N.F.V. e Moreira, L.M. 2005. Características morfogênicas e estruturais do capim xaraés submetido à adubação nitrogenada e desfolhação. Rev Bras Zootecn, 34: 14751482.

Matthew, C., Assuero, S.G., Black, C.K. and Sackville Hamiltom, N.R. 2000. Tiller dynamics of grazed swards. In: Lemaire, G., J. Hodgson, A. Moraes, P.C.F. Carvalho and C. Nabinger (Eds.). Grassland ecophysiology and grazing ecology. CABI Publishing. Wallingford. pp.127-150.

Moreira, L.M., Martuscello, J.A., Fonseca, D.M., Mistura, C., Morais, R.V. e Ribeiro Júnior, J.I. 2009. Perfilhamento, acúmulo de forragem e composição bromatológica do capim-braquiária adubado com nitrogênio. Rev Bras Zootecn, 38: 1675-1684.

Nabinger, C. 2002. Manejo da desfolha. Em: Peixoto, A.M., J.C. Moura e V.P. Faria (Eds.). Anais do $19^{\circ}$ Simpósio sobre Manejo da Pastagem. Tema: Inovações tecnológicas no manejo de pastagens. FEALQ. Piracicaba, SP. pp. 133-158.

Nascimento Júnior, D., Garcez Neto, A.F., Barbosa, R.A. e Andrade, C.M.S. 2002. Fundamentos para o manejo de pastagens: Evolução e atualidade. Em: Pereira, O.G., J.A. Obeid, D.M. Fonseca e D. Nascimento Júnior (Eds.). Simpósio sobre manejo estratégico da pastagem. Anais... UFV. Viçosa. pp. 149-196.
Parsons, A.J., Leaf, E.L., Collett, B. and Stiles, W. 1983. The physiology of grass production under grazing. 1. Characteristics of leaf and carropy photosynthesis of continuously grazed swards. $J$ Appl Ecol, 20: 117-126.

Sbrissia, A.F. e Da Silva, S.C. 2008. Compensação tamanho/densidade populacional de perfilhos em pastos de capim-marandu. Rev Bras Zootecn, 37: 35-47.

Silva, C.C.F., Bonomo, P., Pires, A.J.V., Maranhão, C.M.A., Patês, N.M.S. e Santos, L.C. 2009. Características morfogênicas e estruturais de duas espécies de braquiária adubadas com diferentes doses de nitrogênio. Rev Bras Zootecn, 38: 657-661.

Silveira, M.C.T., Nascimento Júnior, D., Silva, S.C., Euclides, V.P.B., Montagner, D.B., Sbrissia, A.F., Rodrigues, C.S., Sousa, B.M.L., Pena, K.S. and Vilela, H.H. 2010. Morphogenetic and structural comparative characterization of tropical forage grass cultivars under free growth. Sci Agric, 67: 136-142.

Skinner, R.H. and Nelson, C.J. 1995. Elongation of the grass leaf and its relationship to the phyllochron. Crop Sci, 35: 4-10.

UFV. 1999. Universidade Federal de Viçosa. Sistema de análises estatísticas e genéticas SAEG. Universidade Federal de Viçosa. Manual do usuário (versão 8.0). Viçosa. 138 pp.

Archivos de zootecnia vol. 61, núm. 233, p. 102. 